\title{
Some characterizations of constant breadth curves in Euclidean 4-space $E^{4}$
}

\author{
Huseyin Kocayigit and Zennure Cicek \\ Department of Mathematics, Celal Bayar University, Manisa, Turkey \\ Received: 14 August 2015, Revised: 16 September 2015, Accepted: 30 October 2015 \\ Published online: 11 February 2016.
}

\begin{abstract}
In this study, the differential equation characterizations of curves of constant breadth are given in Euclidean 4-space $E^{4}$. Furthermore, a criterion for a curve to be the curve of constant breadth in $E^{4}$ is introduced. As an example, the obtained results are applied to the case that the curvatures $k_{1}, k_{2}, k_{3}$ and are discussed.
\end{abstract}

Keywords: Constant breadth curve, Frenet frame.

\section{Introduction}

Euler introduced the constant breadth curves in 1778 [7]. He considered these special curves in the plane. Later, many geometers have shown increased interest in the properties of plane convex curves. Struik published a brief review of the most important publications on this subject [20]. Also, Ball [1], Barbier [2], Blaschke [3,4] and Mellish [14] investigated the properties of plane curves of constant breadth. A space curve of constant breadth was obtained by Fujiwara by taking a closed curve whose normal plane at a point $P$ has only one more point $Q$ in common with the curve, and for which the distance $d(P, Q)$ is constant $[8]$.

He also defined and studied constant breadth surfaces. Later, Smakal studied the constant breadth space curves [19]. Furthermore, Blaschke considered the notion of curve of constant breadth on the sphere [4]. Moreover, Reuleaux studied the curves of constant breadth and gave the method related to these curves for the kinematics of machinery [16]. Then, constant breadth curves had an importance for engineering sciences and Tanaka used the constant breadth curves in the kinematics design of Com follower systems [21].

Moreover, Köse has presented some concepts for space curves of constant breadth in Euclidean 3-space in [12] and Sezer has obtained the differential equations characterizing space curves of constant breadth and introduced a criterion for these curves [18]. Constant breadth curves in Euclidean 4-space were given by Mağden and Köse [13]. Moreover, constant breath curves have been studied in Minkowski space. Kazaz, Önder and Kocayiğit have studied spacelike curves of constant breadth in Minkowski 4-space [10]. Önder, Kocayiğit and Candan have obtained and studied the differential equations characterizing constant breadth curves in Minkowski 3-space [15]. Furthermore, Kocayiğit and Önder have showed that constant breadth curves are normal curves, helices, and spherical curves in some special cases [11].

In this paper, we study the differential equations characterizing curves of constant breadth in the Euclidean 4-space $E^{4}$. Moreover, we give a criterion characterizing these curves in $E^{4}$. 


\section{Differential equations characterizing curves of constant breadth in $E^{4}$}

Let $(C)$ be a unit speed regular curve in $E^{4}$ with parametrization $\alpha(s): I \subset \mathbb{R} \rightarrow E^{4}$. Denote by $\{\mathbf{T}, \mathbf{N}, \mathbf{B}, \mathbf{E}\}$ the moving Frenet frame along the curve $(C)$ in $E^{4}$. Then, the following Frenet formulate are given,

$$
\left[\begin{array}{c}
\mathbf{T}^{\prime} \\
\mathbf{N}^{\prime} \\
\mathbf{B}^{\prime} \\
\mathbf{E}^{\prime}
\end{array}\right]=\left[\begin{array}{cccc}
0 & k_{1} & 0 & 0 \\
-k_{1} & 0 & k_{2} & 0 \\
0 & -k_{2} & 0 & k_{3} \\
0 & 0 & -k_{2} & 0
\end{array}\right]\left[\begin{array}{c}
\mathbf{T} \\
\mathbf{N} \\
\mathbf{B} \\
\mathbf{E}
\end{array}\right]
$$

where $k_{1}, k_{2}$ and $k_{3}$ are the first, second and third curvatures of the curve $(C)$, respectively [9].

Definition 1. Let $(C)$ be a unit speed regular curve in $E^{4}$ with position vector $\alpha(s)$. If $(C)$ has parallel tangents $\mathbf{T}$ and $\mathbf{T}^{*}$ in opposite direction at the opposite points and of the curve and if the distance between these points is always constant then is called a curve of constant breadth in $E^{4}$. Moreover, a pair of curves $(C)$ and $\left(C^{*}\right)$ for which the tangents at the corresponding points are parallel and in opposite directions and the distance between these points is always constant is called a curve pair of constant breadth in $E^{4}$.

Let now $(C)$ and $\left(C^{*}\right)$ be a pair of unit speed curves in $E^{4}$ with position vector $\alpha(s)$ and $\alpha^{*}\left(s^{*}\right)$, where $s$ and $s^{*}$ are arc length parameters of the curves, respectively. Let $(C)$ and $\left(C^{*}\right)$ have parallel tangents in opposite directions at opposite points. Then the curve $\left(C^{*}\right)$ may be represented by the equation

$$
\alpha^{*}(s)=\alpha(s)+m_{1}(s) \mathbf{T}(s)+m_{2}(s) \mathbf{N}(s)+m_{3}(s) \mathbf{B}(s)+m_{4}(s) \mathbf{E}(s)
$$

where $m_{i}(s), 1 \leq i \leq 4$ are the differentiable functions of $s$ which is the arc length of $(C)$. Differentiating this equation with respect to $s$ and using the Frenet formulate we obtain

$$
\frac{\alpha^{*}(s)}{d s}=\mathbf{T}^{*} \frac{d s^{*}}{d s}=\left(1+\frac{d m_{1}}{d s}-m_{2} k_{1}\right) \mathbf{T}+\left(m_{1} k_{1}+\frac{d m_{2}}{d s}-m_{3} k_{2}\right) \mathbf{N}+\left(m_{2} k_{2}+\frac{d m_{3}}{d s}-m_{4} k_{3}\right) \mathbf{B}+\left(m_{3} k_{3}+\frac{d m_{4}}{d s}\right) \mathbf{E}
$$

Since $\mathbf{T}=-\mathbf{T}^{*}$ at the corresponding points of $(C)$ and $\left(C^{*}\right)$, we have

$$
\left\{\begin{array}{l}
\left(1+\frac{d m_{1}}{d s}-m_{2} k_{1}\right)=-\frac{d s^{*}}{d s} \\
\left(m_{1} k_{1}+\frac{d m_{2}}{d s}-m_{3} k_{2}\right)=0 \\
\left(m_{2} k_{2}+\frac{d m_{3}}{d s}-m_{4} k_{3}\right)=0 \\
\left.m_{3} k_{3}+\frac{d m_{4}}{d s}\right)=0 .
\end{array}\right.
$$

It is well known that the curvature of $(C)$ is $\lim (\Delta \varphi / \Delta s)=(d \varphi / d s)=k_{1}(s)$, where $\varphi=\int_{0}^{s} k_{1}(s) d s$ is the angle between the tangent of the curve $(C)$ and a given fixed direction at the point $\alpha(s)$. Then from (2) we have the following system

$$
\begin{aligned}
& m_{1}^{\prime}=m_{2}-f(\varphi), m_{2}^{\prime}=m_{3} \rho k_{2}, \\
& m_{3}^{\prime}=m_{4} \rho k_{3}-m_{2} \rho k_{2}, m_{4}^{\prime}=-m_{3} \rho k_{3} .
\end{aligned}
$$

Here and after we will use $\left(^{\prime}\right)$ to show the differentiation with respect to $\varphi$. In $(3), f(\varphi)=\rho+\rho^{*}$ and, $\rho=\frac{1}{k_{1}}$ and $\rho^{*}=\frac{1}{k_{1}^{*}}$ denote the radius of curvatures at the points $\alpha$ and $\alpha^{*}$, respectively. From (3) eliminating $m_{2}, m_{3}$ and $m_{4}$ their derivatives we have the following differential equation

$$
\frac{d}{d \varphi}\left[\frac{d}{d \varphi}\left[\frac{1}{\rho k_{2}}\left(\frac{d^{2} m_{1}}{d \varphi^{2}}+m_{1}\right)\right]+\frac{k_{2}}{k_{3}} \frac{d m_{1}}{d \varphi}\right]+\frac{k_{2}}{k_{3}}\left(\frac{d^{2} m_{1}}{d \varphi^{2}}+m_{1}\right)+\frac{d}{d \varphi}\left[\frac{1}{\rho k_{2}} \frac{d}{d \varphi}\left(\frac{1}{\rho k_{2}} \frac{d f}{d \varphi}\right)+\frac{k_{2}}{k_{3}} f\right]+\frac{k_{2}}{k_{3}} \frac{d f}{d \varphi}=0 .
$$


Then we can give the following theorem.

Theorem 1. The general differential equation characterizing space curves of constant breadth in $E^{4}$ is given by (4).

Let now consider the system (3) again. The distance $d$ between the opposite points $\alpha$ and $\alpha^{*}$ is the breadth of the curves and is constant, that is,

$$
d^{2}=\|\mathbf{d}\|^{2}=\left\|\alpha^{*}-\alpha\right\|^{2}=m_{1}^{2}+m_{2}^{2}+m_{3}^{2}+m_{4}^{2}=\text { const } .
$$

Then the system (3) may be written as follows:

$$
\begin{aligned}
& m_{2}=f(\varphi), m_{2}^{\prime}=m_{3} \rho k_{2}, m_{3}^{\prime}=m_{4} \rho k_{3}-m_{2} \rho k_{2}, \\
& m_{4}^{\prime}=-m_{3} \rho k_{3}, m_{1}=0 .
\end{aligned}
$$

or

$$
\begin{aligned}
& m_{1}^{\prime}=m_{2}, m_{2}^{\prime}=-m_{1}+m_{3} \rho k_{2}, \\
& m_{3}^{\prime}=m_{4} \rho k_{3}-m_{2} \rho k_{2}, m_{4}^{\prime}=-m_{3} \rho k_{3} .
\end{aligned}
$$

which are the systems describing the curve $(C)$.

Let us consider the system (7) with special chosen $m_{1}=$ const. . Here, eliminating first $m_{1}, m_{2}, m_{3}$ and their derivatives, and then $m_{1}, m_{2}, m_{4}$ and their derivatives, respectively, we obtain the following linear differential equations of second order

$$
\begin{cases}\left(\rho k_{3}\right) m_{4}^{\prime \prime}-\left(\rho k_{3}\right)^{\prime} m_{4}^{\prime}+\left(\rho k_{3}\right)^{3} m_{4}=0, & \rho k_{2} \neq 0, \\ \left(\rho k_{3}\right) m_{3}^{\prime \prime}-\left(\rho k_{3}\right)^{\prime} m_{3}^{\prime}+\left(\rho k_{3}\right)^{3} m_{3}=0, & \rho k_{3} \neq 0 .\end{cases}
$$

By changing the variable $\varphi$ of the form $\xi=\int_{0}^{\varphi} \rho(t) k_{3}(t) d t$, these equations can be transformed into the following differential equations with constant coefficients,

$$
\frac{d^{2} m_{4}}{d \xi^{2}}+m_{4}=0 \text { and } \frac{d^{2} m_{3}}{d \xi^{2}}+m_{3}=0
$$

respectively [5]. Then, the general solutions of the differential equations (9) are

$$
\left\{\begin{array}{l}
m_{3}=A \cos \left(\int_{0}^{\varphi} \rho k_{3} d t\right)+B \sin \left(\int_{0}^{\varphi} \rho k_{3} d t\right), \\
m_{4}=C \cos \left(\int_{0}^{\varphi} \rho k_{3} d t\right)+D \sin \left(\int_{0}^{\varphi} \rho k_{3} d t\right) .
\end{array}\right.
$$

respectively, where $A, B, C$ and $D$ are real constants. Substituting (10) into (7), we obtain $A=-D, B=C$, and so, the set of the solutions of the system (7), in the form

$$
\left\{\begin{array}{c}
m_{1}=c=\text { const. } m_{2}=0 \\
m_{3}=A \cos \int_{0}^{\varphi} \rho k_{3} d t+B \sin \int_{0}^{\varphi} \rho k_{3} d t \\
m_{4}=B \cos \int_{0}^{\varphi} \rho k_{3} d t-A \sin \int_{0}^{\varphi} \rho k_{3} d t .
\end{array}\right\}
$$

Thus the equation (1) is described and since $d^{2}=\left\|\alpha^{*}-\alpha\right\|^{2}=$ const., from (11) the breadth of the curve is $d^{2}=c^{2}+A^{2}+B^{2}$.

Now, let us return to the system (6) with $m_{1}=0$. By changing the variable $\varphi$ of the form $u=\int_{0}^{\varphi} \mu(t) d t, \mu=\rho k_{3}$ and 
eliminating $m_{1}, m_{2}, m_{4}$ and their derivatives we have the linear differential equation

$$
\frac{d^{2} m_{3}}{d u^{2}}+m_{3}=-\frac{d}{d u}\left(\frac{k_{2}}{k_{3}} m_{2}\right)
$$

which has the following solution

$$
m_{3}=A_{1} \cos \int_{0}^{\varphi} \rho k_{3} d t+B_{1} \sin \int_{0}^{\varphi} \rho k_{3} d t-\int_{0}^{\varphi} \cos [u(\varphi)-u(t)] \rho k_{2} f(t) d t
$$

Then, the general solution of the system (6) is

$$
\left\{\begin{array}{l}
m_{1}=0 \\
m_{2}=f(\varphi) \\
m_{3}=A_{1} \cos \int_{0}^{\varphi} \rho k_{3} d t+B_{1} \sin \int_{0}^{\varphi} \rho k_{3} d t-\int_{0}^{\varphi} \cos [u(\varphi)-u(t)] \rho k_{2} f(t) d t \\
m_{4}=B_{1} \cos \int_{0}^{\varphi} \rho k_{3} d t-A_{1} \sin \int_{0}^{\varphi} \rho k_{3} d t+\int_{0}^{\varphi} \sin [u(\varphi)-u(t)] \rho k_{2} f(t) d t
\end{array}\right.
$$

which determines the constant breadth curve in (1) where $A_{1}, B_{1}$ are real constants.

Furthermore, in this case, i.e., $m_{1}=0$, from (4) we have the following differential equation

$$
\frac{d}{d \varphi}\left[\frac{1}{\rho k_{3}} \frac{d}{d \varphi}\left(\frac{1}{\rho k_{2}} \frac{d f}{d \varphi}\right)+\frac{k_{2}}{k_{3}} f\right]+\frac{k_{2}}{k_{3}} \frac{d f}{d \varphi}=0
$$

By changing the variable $\varphi$ of the form $w=\int_{0}^{\varphi} \rho k_{2} d \varphi,(15)$ becomes

$$
\frac{d}{d w}\left[\frac{k_{2}}{k_{3}}\left(\frac{d^{2} f}{d w^{2}}+f\right)\right]+\frac{k_{3}}{k_{2}} \frac{d f}{d w}=0 .
$$

which also determines the constant breadth curve in (1).

So far we have dealt with a pair of space curves having parallel tangent in opposite directions at corresponding points. Now let us consider a simple closed unit speed space curve $(C)$ in $E^{4}$ for which the normal plane of every point $P$ on the curve meets the curve of a single opposite point $Q$ other than $P$. Then, we may give the following theorem concerning the space curves of constant breadth in $E^{4}$.

Theorem 2. Let $(C)$ be a closed space curve in $E^{4}$ having parallel tangents in opposite directions at the opposite points of the curve. If the chord joining the opposite points of $(C)$ is a double-normal, then $(C)$ has constant breadth, and conversely, if $(C)$ is a curve of constant breadth in $E^{4}$ then every normal of $(C)$ is a double-normal.

Proof. Let the vector $\mathbf{d}=\alpha^{*}-\alpha=m_{1} \mathbf{T}+m_{2} \mathbf{N}+m_{3} \mathbf{B}+m_{4} \mathbf{E}$ be a double-normal of $(C)$ where $m_{1}, m_{2}, m_{3}$ and $m_{4}$ are the functions of $s$, the arc length parameter of the curve. Then we get $\left\langle\mathbf{d}, \mathbf{T}^{*}\right\rangle=-\langle\mathbf{d}, \mathbf{T}\rangle=m_{1}=0$. Thus from (2) we have

$$
m_{2} \frac{d m_{2}}{d s}+m_{3} \frac{d m_{3}}{d s}+m_{4} \frac{d m_{4}}{d s}=0 .
$$

It follows that $m_{2}^{2}+m_{3}^{2}+m_{4}^{2}=$ constant, i.e., the breadth of $(C)$ is constant.

Conversely, if $\|\mathbf{d}\|^{2}=m_{1}^{2}+m_{2}^{2}+m_{3}^{2}+m_{4}^{2}=$ constant then as shown, $m_{1}=0$. This means that $\mathbf{d}$ is perpendicular to $\mathbf{T}$ and $\mathbf{T}^{*}$. So, $\mathbf{d}$ is the double-normal of $(C)$.

A simple closed curve having parallel tangents in opposite directions at opposite points may be represented by the 
system (14). In this case a pair of opposite points of the curve is $\left(\alpha^{*}(\varphi), \alpha(\varphi)\right)$ for $\varphi$, where $0 \leq \varphi \leq 2 \pi$. Since $(C)$ is a simple closed curve we get $\alpha^{*}(0)=\alpha^{*}(2 \pi)$. Hence from (14) we have

$$
\int_{0}^{2 \pi} \rho k_{3} d t=2 n \pi, \quad(n \in \mathbb{Z}) .
$$

Using the equality $d s=\rho d \varphi$, this formula may be given as $\int_{C} k_{3} d s=2 n \pi$,

$(n \in \mathbb{Z})$. This says that the integral third curvature of $(C)$ is zero. So, we can give the following corollary.

Corollary 1. The total third curvature of a simple closed curve $(C)$ of constant breadth is $2 n \pi, n \in \mathbb{Z}$.

Furthermore, if we take $\frac{k_{2}}{k_{3}}=a=$ constant, then from (16) we have

$$
\frac{d^{3} f}{d w^{3}}+K \frac{d f}{d w}=0
$$

where $K=1+\frac{1}{a^{2}}$. If we assume $K \neq \pm 1$, the general solution of (19) is

$$
f=A_{2} \sin \int_{0}^{\varphi} K \rho k_{2} d t+B_{2} \cos \int_{0}^{\varphi} K \rho k_{2} d t+C_{1} .
$$

where $A_{2}, B_{2}$ and $C_{1}$ are real constants. Since $(C)$ is a simple closed curve, i.e., $\alpha^{*}(0)=\alpha^{*}(2 \pi)$, from (20) it follows,

$$
\int_{0}^{\varphi} K \rho k_{2} d t=2 n \pi, \quad(n \in \mathbb{Z}) .
$$

Using the equality $d s=\rho d \varphi$, this formula may be given as $\int_{C} k_{2} d s=2 \frac{n}{K} \pi,(K, n \in \mathbb{Z})$. This says that the integral second curvature of $(C)$ is $2 \frac{n}{K} \pi,(K, n \in \mathbb{Z})$. So, we can give the following corollary.

Corollary 2. The total second curvature of a simple closed curve $(C)$ of constant breadth with $a=k_{2} / k_{3}=$ constant is $2 \frac{n}{K} \pi$, where $n \in \mathbb{Z}$ and $K=1+\frac{1}{a^{2}}$.

\section{A criterion for curves of constant breadth in $E^{4}$}

Let us assume that $(C)$ is a curve of constant breadth in $E^{4}$ and $\alpha(s)$ denotes the position vector of a generic point of the curve. If $(C)$ is a closed curve, the position vector $\alpha(s)$ must be a periodic function of period $\omega=2 \pi$, where $\omega$ is the total length of $(C)$. Then the curvatures $k_{1}(s), k_{2}(s)$ and $k_{3}(s)$ are also periodic of the same period. However, periodicity of the curvatures and closeness of the curve are not sufficient to guarantee that a space curve is a constant breadth curve in $E^{4}$. That is, if a curve is closed curve (periodic), it may be the curve of constant breadth or not. Therefore, to guarantee that a curve is a constant breadth curve, we may use the system (7) characterizing a curve of constant breadth and follow the similar way given in [6].

For this purpose, first let us consider the following Frenet formulas at a generic point on the curve $(C)$,

$$
\frac{d \mathbf{T}}{d s}=k_{1} \mathbf{N}, \frac{d \mathbf{N}}{d s}=-k_{1} \mathbf{T}+k_{2} \mathbf{B}, \frac{d \mathbf{B}}{d s}=-k_{2} \mathbf{N}+k_{3} \mathbf{E}, \frac{d \mathbf{E}}{d s}=-k_{3} \mathbf{B} .
$$

Writing the formulas (22) in terms of $\varphi$ and allowing for $\frac{d \varphi}{d s}=k_{1}=\frac{1}{\rho}$ we have

$$
\frac{d \mathbf{T}}{d \varphi}=\mathbf{N}, \frac{d \mathbf{N}}{d \varphi}=-\mathbf{T}+\rho k_{2} \mathbf{B}, \frac{d \mathbf{B}}{d \varphi}=-\rho k_{2} \mathbf{N}+\rho k_{3} \mathbf{E}, \frac{d \mathbf{E}}{d \varphi}=-\rho k_{3} \mathbf{B}
$$


Furthermore we can write the Frenet vectors $\mathbf{T}, \mathbf{N}, \mathbf{B}, \mathbf{E}$ in the coordinate forms as follows

$$
\mathbf{T}=\sum_{i=1}^{4} t_{i} \mathbf{e}_{\mathbf{i}}, \mathbf{N}=\sum_{i=1}^{4} n_{i} \mathbf{e}_{\mathbf{i}}, \mathbf{B}=\sum_{i=1}^{4} b_{i} \mathbf{e}_{\mathbf{i}}, \mathbf{E}=\sum_{i=1}^{4} \varepsilon_{i} \mathbf{e}_{\mathbf{i}}
$$

Since $\{\mathbf{T}, \mathbf{N}, \mathbf{B}, \mathbf{E}\}$ is the orthonormal base in $E_{1}^{4}$, putting (24) and their derivatives into (23), we have the systems of linear differential equations

$$
\left\{\begin{array}{llll}
\frac{d t_{1}}{d \varphi}=n_{1}, & \frac{d t_{2}}{d \varphi}=n_{2}, & \frac{d t_{3}}{d \varphi}=n_{3}, & \frac{d t_{4}}{d \varphi}=n_{4} \\
\frac{d n_{1}}{d \varphi}=-t_{1}+\rho k_{2} b_{1}, & \frac{d n_{2}}{d \varphi}=-t_{2}+\rho k_{2} b_{2}, & \frac{d n_{3}}{d \varphi}=-t_{3}+\rho k_{2} b_{3}, & \frac{d n_{4}}{d \varphi}=-t_{4}+\rho k_{2} b_{4} \\
\frac{d b_{1}}{d \varphi}=\rho k_{3} \varepsilon_{1}-\rho k_{2} n_{1}, & \frac{d b_{2}}{d \varphi}=\rho k_{3} \varepsilon_{2}-\rho k_{2} n_{2}, & \frac{d b_{3}}{d \varphi}=\rho k_{3} \varepsilon_{3}-\rho k_{2} n_{3}, & \frac{d b_{4}}{d \varphi}=\rho k_{3} \varepsilon_{4}-\rho k_{2} n_{4} \\
\frac{d \varepsilon_{1}}{d \varphi}=-\rho k_{3} b_{1}, & \frac{d \varepsilon_{2}}{d \varphi}=-\rho k_{3} b_{2}, & \frac{d \varepsilon_{3}}{d \varphi}=-\rho k_{3} b_{3}, & \frac{d \varepsilon_{4}}{d \varphi}=-\rho k_{3} b_{4} .
\end{array}\right\}
$$

From (25), we find that $\left\{t_{1}, n_{1}, b_{1}, \varepsilon_{1}\right\},\left\{t_{2}, n_{2}, b_{2}, \varepsilon_{2}\right\},\left\{t_{3}, n_{3}, b_{3}, \varepsilon_{3}\right\}$ and $\left\{t_{4}, n_{4}, b_{4}, \varepsilon_{4}\right\}$ are four independent solutions of the following system of differential equations:

$$
\frac{d \psi_{1}}{d \varphi}=\psi_{2}, \frac{d \psi_{2}}{d \varphi}=-\psi_{1}+\rho k_{2} \psi_{3}, \frac{d \psi_{3}}{d \varphi}=\rho k_{3} \psi_{4}-\rho k_{2} \psi_{2}, \frac{d \psi_{4}}{d \varphi}=-\rho k_{3} \psi_{3} .
$$

If the curve $(C)$ is the curve of constant breadth, then the systems (7) and (26) must be the same system. So, we observe that $\psi_{1}=m_{1}, \psi_{2}=m_{2}, \psi_{3}=m_{3}, \psi_{4}=m_{4}$. For brevity, we can write (7) or (26) in the form

$$
\frac{d \psi}{d \varphi}=A(\varphi) \psi
$$

where

$$
\psi=\left[\begin{array}{l}
m_{1} \\
m_{2} \\
m_{3} \\
m_{4}
\end{array}\right], A(\varphi)=\left[\begin{array}{cccc}
0 & 1 & 0 & 0 \\
-1 & 0 & \rho k_{2} & 0 \\
0 & -\rho k_{2} & 0 & \rho k_{3} \\
0 & 0 & -\rho k_{3} & 0
\end{array}\right]
$$

Obviously, (27) is a special case of the general linear differential equations abbreviated to the form

$$
\left\{\begin{array}{l}
\frac{d \psi}{d t}=A(t) \psi \\
\varphi=\left[\begin{array}{c}
m_{1} \\
m_{2} \\
\vdots \\
m_{N}
\end{array}\right], A(t)=\left[\begin{array}{cccc}
a_{11} & a_{12} & \cdots & a_{1 n} \\
a_{21} & a_{22} & \cdots & a_{2 n} \\
\vdots & \vdots & \ddots & \vdots \\
a_{n 1} & a_{n 2} & \cdots & a_{n n}
\end{array}\right],(4 \leq n) .
\end{array}\right.
$$

where $a_{i j}(t)$ are assumed to be continuous and periodic of period $\omega$ (See [6,17]). Let the initial conditions be $\psi_{i}(0)=x_{i}$, $(i=1,2, \ldots, n)$. Let us take $x=\left[x_{1}, x_{2}, \ldots, x_{n}\right]^{T}$ and

$$
\psi(t, x)=\left[m_{1}(t, x) m_{2}(t, x) \ldots m_{n}(t, x)\right]^{T}
$$

Then the equation (28) may be written in the form $\frac{d \psi}{d t}=A(t) \psi, \psi(0)=x$ as is well known from $[6]$, the solution $\psi(t, x)$ of this equation is periodic of period $\omega$, if

$$
\int_{0}^{\omega} A(\xi) \psi(\xi, x) d \xi=0
$$


and

$$
\left\{\begin{array}{l}
\psi(t, x)=\{E+M(t)\} x,(E=\text { unit matrix }), \\
M(t)=I A(t)+I^{(2)} A(t)+\ldots+I^{(n)} A(t)+\ldots \\
(I A)(t)=I^{(I)} A(t)=\int_{0}^{t} A(\xi) d \xi \\
\left(I^{(n)} A\right)(t)=\int_{0}^{t} A(\xi)\left(I^{(n-1)} A\right)(\xi) d \xi, n>1 .
\end{array}\right.
$$

Furthermore, the following theorem is given in [6]:

Theorem 3. The equations $\frac{d \psi}{d t}=A(t) \psi$ possess a non-vanishing periodic solution of period $\omega$, if and only if det $(M(\omega))=$ 0 . In particular, in order that the equations $\frac{d \psi}{d t}=A(t) \psi$ possess linearly independent periodic solutions of period $\omega$, the necessary and sufficient condition is that $M(\omega)$ be a zero matrix.

Now, let us apply this theorem to the system (27). If $M(\omega)=0$, there exist the unit vector functions $\mathbf{T}, \mathbf{N}, \mathbf{B}, \mathbf{E}$ of period $\omega$, such that each set of functions $\left\{t_{i}, n_{i}, b_{i}, \varepsilon_{i}\right\},(i=1,2,3,4)$ form a solution of the equation (27) corresponding to the initial conditions $\left(A_{i}, B_{i}, C_{i}, D_{i}\right)$. The curve $(C)$ can be described as follows

$$
\alpha(s)=\int_{0}^{s} \mathbf{T}(s) d s \quad \text { or } \quad \alpha(\varphi)=\int_{0}^{\varphi} \rho(\varphi) \mathbf{T}(\varphi) d(\varphi) .
$$

Here, to find $\mathbf{T}$, we can make use of the equation

$$
\left[\begin{array}{c}
t_{i} \\
n_{i} \\
b_{i} \\
\varepsilon_{i}
\end{array}\right]=\{E+M(\varphi)\}\left[\begin{array}{c}
A_{i} \\
B_{i} \\
C_{i} \\
D_{i}
\end{array}\right],(i=1,2,3,4) .
$$

which is established by (29). If we take the initial conditions as $t_{i}(0)=A_{i}, n_{i}(0)=B_{i}, b_{i}(0)=C_{i}, \varepsilon_{i}(0)=D_{i},(i=1,2,3,4)$ such that $\left(A_{1}, A_{2}, A_{3}, A_{4}\right),\left(B_{1}, B_{2}, B_{3}, B_{4}\right),\left(C_{1}, C_{2}, C_{3}, C_{4}\right),\left(D_{1}, D_{2}, D_{3}, D_{4}\right)$ form an orthonormal frame, then from (30) we obtain

$$
t_{i}=\left(1+m_{11}\right) A_{i}+m_{12} B_{i}+m_{13} C_{i}+m_{14} D_{i} ; \quad(i=1,2,3,4) .
$$

When the curve $(C)$ is a curve of constant breadth, which is also periodic of period $\omega$, it is clear that

$$
\int_{0}^{\omega} \rho t_{i} d \varphi=0
$$

Hence, form (31) and (32), we have

$$
A_{i} \int_{0}^{\omega} \rho\left(1+m_{11}\right) d \varphi+B_{i} \int_{0}^{\omega} \rho m_{12} d \varphi+C_{i} \int_{0}^{\omega} \rho m_{13} d \varphi+D_{i} \int_{0}^{\omega} \rho m_{14} d \varphi=0 ;(i=1,2,3,4)
$$

Since the coefficient determinant $\Delta \neq 0$ in this system, we obtain the equalities

$$
\int_{0}^{\omega} \rho\left(1+m_{11}\right) d \varphi=0=\int_{0}^{\omega} \rho m_{12} d \varphi=\int_{0}^{\omega} \rho m_{13} d \varphi=\int_{0}^{\omega} \rho m_{14} d \varphi
$$

which are the conditions for a curve to be constant breadth curve in $E^{4}$. Here, we can take the period $\omega=2 \pi$ because of $0 \leq \varphi \leq 2 \pi$. Thus we establish the following corollary.

Corollary 3. Let $(C)$ be a regular curve in $E^{4}$ such that $\rho(\varphi)>0, k_{2}(\varphi)$ and $k_{3}(\varphi)$ are continuous periodic functions of period $\omega$. Then $(C)$ is a curve of constant breadth, and also periodic of period $\omega$, if and only if

$$
M(\omega)=0, \int_{0}^{\omega} \rho\left(1+m_{11}\right) d \varphi=0=\int_{0}^{\omega} \rho m_{12} d \varphi=\int_{0}^{\omega} \rho m_{13} d \varphi=\int_{0}^{\omega} \rho m_{14} d \varphi .
$$


holds, where

$$
\left\{\begin{aligned}
M(t) & =I A(t)+I^{(2)} A(t)+\ldots+I^{(n)} A(t)+\ldots \\
A(t)= & {\left[\begin{array}{cccc}
0 & 1 & 0 & 0 \\
-1 & 0 & \rho k_{2} & 0 \\
0 & -\rho k_{2} & 0 & \rho k_{3} \\
0 & 0 & -\rho k_{3} & 0
\end{array}\right] }
\end{aligned}\right.
$$

and $m_{i j}(t)$ are the entries of the matrix $M(t)$.

By means of (29) and (35), the matrix $M(t)$ can be constructed and each $m_{i j}$ involves infinitely many integrations. Hence, we can write the conditions (34) in the following forms:

$$
\left\{\begin{array}{l}
\int_{0}^{\omega} \rho(\varphi) d \varphi-\int_{0}^{\omega} \int_{0}^{r} \int_{0}^{s} \rho(\varphi) d s d t d \varphi+\int_{0}^{\omega} \int_{0}^{\phi} \int_{0}^{p} \int_{0}^{r} \int_{0}^{s} \rho(\varphi)[1+\lambda(p) \lambda(s)] d t d s d r d p d \varphi-\ldots=0 \\
\int_{0}^{\omega} \int_{0}^{s} \rho(\varphi) d t d \varphi-\int_{0}^{\omega} \int_{0}^{p} \int_{0}^{r} \int_{0}^{s} \rho(\varphi)[1+\lambda(t) \lambda(s)] d t d s d r d \varphi+\ldots=0 \\
\int_{0}^{\omega} \int_{0}^{r} \int_{0}^{s} \rho(\varphi) \lambda(t) d t d s d \varphi-\int_{0}^{\omega} \int_{0}^{\phi} \int_{0}^{p} \int_{0}^{r} \int_{0}^{s} \rho(\varphi)[\lambda(t)+\lambda(p)\{\lambda(t) \lambda(s)+\mu(t) \mu(s)\}] d t d s d r d p d \varphi+\ldots=0 \\
\int_{0}^{\omega} \int_{0}^{p} \int_{0}^{r} \int_{0}^{s} \rho(\varphi) \lambda(s) \mu(t) d t d s d r d \varphi-\int_{0}^{\omega} \int_{0}^{q} \int_{0}^{\phi} \int_{0}^{p} \int_{0}^{r} \int_{0}^{s} \rho(\varphi) \lambda(p) \mu(t)[1+\lambda(t) \lambda(s)+\mu(t) \mu(s)] d t d s d p d \phi d \varphi+\ldots=0 .
\end{array}\right.
$$

where $\lambda(\xi)=p(\xi) k_{2}(\xi), \mu(\xi)=p(\xi) k_{3}(\xi)$.

Example 1. Let us consider the special case $\rho=$ const., $k_{2}=$ const. and $k_{3}=$ const. In this case, from (33), we have

$$
\left\{\begin{array}{l}
\omega-\frac{\omega^{3}}{3 !}+\left(1+\rho^{2} k_{2}^{2}\right) \frac{\omega^{5}}{5 !}-\varepsilon\left(1+\rho^{2} k_{2}^{2}\right)^{2} \frac{\omega^{7}}{7 !} \ldots=0 \\
\frac{\omega^{2}}{2 !}-\left(1+\rho^{2} k_{2}^{2}\right) \frac{\omega^{4}}{4 !}+\left(1+\rho^{2} k_{2}^{2}\right)^{2} \frac{\omega^{6}}{6 !}-\ldots=0 \\
k_{2}\left[\frac{\omega^{3}}{3 !}-\left(1+\rho^{2} k_{2}^{2}+\rho^{2} k_{3}^{2}\right) \frac{\omega^{5}}{5 !}+\left(1+\rho^{2} k_{2}^{2}+\rho^{2} k_{3}^{2}\right)^{2} \frac{\omega^{7}}{7 !}-\ldots\right]=0 \\
k_{2} k_{3}\left[\frac{\omega^{4}}{4 !}-\left(1+\rho^{2} k_{2}^{2}+\rho^{2} k_{3}^{2}\right) \frac{\omega^{6}}{6 !}+\ldots\right]=0
\end{array}\right.
$$

or

$$
\left\{\begin{array}{l}
\rho^{2} k_{2}^{2}\left(1+\rho^{2} k_{2}^{2}\right)^{\frac{1}{2}} \omega+\sin \left[\left(1+\rho^{2} k_{2}^{2}\right)^{\frac{1}{2}} \omega\right]=0, \\
\cos \left[\left(1+\rho^{2} k_{2}^{2}\right)^{\frac{1}{2}} \omega\right]=1 \text { or }\left(1+\rho^{2} k_{2}^{2}\right)^{\frac{1}{2}} \omega=2 k \pi, k \in \mathbb{Z} \\
k_{2}\left[\left(1+\rho^{2} k_{2}^{2}+\rho^{2} k_{3}^{2}\right)^{\frac{1}{2}} \omega-\sin \left[\left(1+\rho^{2} k_{2}^{2}+\rho^{2} k_{3}^{2}\right)^{\frac{1}{2}} \omega\right]\right]=0, \\
k_{2} k_{3}\left[-1+\left(1+\rho^{2} k_{2}^{2}+\rho^{2} k_{3}^{2}\right) \frac{\omega^{2}}{2}+\cos \left[\left(1+\rho^{2} k_{2}^{2}+\rho^{2} k_{3}^{2}\right)^{\frac{1}{2}} \omega\right]\right]=0 .
\end{array}\right.
$$

where $\omega=2 k \pi$. It is seen that all of the equalities (37) or (38) are satisfied simultaneously, if and only if $\rho k_{2}=0$, $\rho k_{3}=0$ that is, $\rho=$ const. $>0$ and $k_{2}, k_{3}=0$. Therefore, only ones with $\rho=$ const. $>0$ and $k_{2}, k_{3}=0$ of the curves with and are curves of constant breadth, which are circles in $E^{4}$.

Now let us construct the relation characterizing these circles. Since $\rho k_{2}, \rho k_{3}=0$ system (7) becomes

$$
m_{1}^{\prime}=m_{2}, m_{2}^{\prime}=-m_{1}, m_{3}^{\prime}=0, m_{4}^{\prime}=0 \text {. }
$$

From (39), the equations with the unknowns $m_{1}, m_{2}$ and $m_{3}$ can be written as follows

$$
m_{1}^{\prime \prime}+m_{1}=0, m_{2}^{\prime \prime}+m_{2}=0, m_{3}^{\prime}=0, m_{4}^{\prime}=0 .
$$

The general solution of $(40)$ is

$$
\left\{\begin{array}{l}
m_{1}=A_{3} \cos (\varphi)+B_{3} \sin (\varphi) \\
m_{2}=C_{2} \cos (\varphi)+D_{1} \sin (\varphi) \\
m_{3}=L_{1} \\
m_{4}=L_{2}
\end{array}\right.
$$


where $A_{3}, B_{3}, C_{2}, D_{1}, L_{1}$ and $L_{2}$ are arbitrary constants. Replacing (41) into (39), we have $A_{3}=-D_{1}, B_{3}=C_{2}$ and thus we get

$$
\left\{m_{1}=A_{3} \cos (\varphi)+B_{3} \sin (\varphi), m_{2}=B_{3} \cos (\varphi)-A_{3} \sin (\varphi), m_{3}=L_{1}, m_{4}=L_{2}\right\} .
$$

which is the solution set of the system (40). Consequently, replacing (42) into (1), we obtain the equation

$$
\alpha^{*}(\varphi)=\alpha(\varphi)+\left(A_{3} \cos (\varphi)+B_{3} \sin (\varphi)\right) \mathbf{T}+\left(B_{3} \cos (\varphi)-A_{3} \sin (\varphi)\right) \mathbf{N}+L_{1} \mathbf{B}+L_{2} \mathbf{E}
$$

which represents the circles with the diameter $d=\left\|\alpha^{*}-\alpha\right\|=\left(A_{3}^{2}+B_{3}^{2}+L_{1}^{2}+L_{2}^{2}\right)^{\frac{1}{2}}$. In this case, a pair of opposite points of the curve is $\left(\alpha^{*}(\varphi), \alpha(\varphi)\right)$ for $\varphi$ in $0 \leq \varphi \leq 2 \pi$.

\section{Conclusion}

In the characterizations and determinations of the special curves and curve pair, the differential equations have an important role. A differential equation or a system of differential equations with respect to the curvatures can determinate the special curves or curve pairs. In this paper, the differential equations characterizing the curves of constant breadth in $E^{4}$ are studied. Furthermore, a criterion for a space curve to be the curve of constant breadth in $E^{4}$ is given.

\section{References}

[1] Ball, N. H., On Ovals, American Mathematical Monthly, 37(7) (1930), 348-353.

[2] Barbier, E., Note sur le probleme de l'aiguille et le jeu du joint couvert. J. Math. Pures Appl., II. Ser. 5, 273-286 (1860).

[3] Blaschke, W., Konvexe bereiche gegebener konstanter breite und kleinsten inhalts, Math. Annalen, 76 (1915), 504-513.

[4] Blaschke, W., Einige Bemerkungen über Kurven und Flächen von konstanter Breite. Ber. Verh. sächs. Akad. Leipzig, 67, 290-297 (1915).

[5] Breuer, S., Gottlieb, D., The Reduction of Linear Ordinary Differential Equations to Equations with Constant Coefficients, J. Math. Anal. Appl., 32 (1970), no. 1, 62-76.

[6] Chung, H.C., A Differential-Geometric Criterion for a Space Curve to be Closed, Proc. Amer. Math. Soc. 83 (1981), no. 2, $357-361$.

[7] Euler, L., De Curvis Triangularibus, Acta Acad. Petropol., (1778), (1780), 3-30.

[8] Fujivara, M., On space curves of constant breadth, Tohoku Math., J., 5 (1914), 180-184.

[9] Hacisalihoğlu, H.H., Diferensiyel Geometri, İnönü University Science and Art Faculty Press, 1983.

[10] Kazaz, M., Önder, M., Kocayiğit H., Spacelike curves of constant breadth in Minkowski 4-space, Int. Journal of Math. Analysis, 2 (2008), no. 22, 1061-1068.

[11] Kocayiğit, H., Önder, M., Spacelike curves of constant breadth in Minkowski 3-space, Annali di Matematica, 192 (5), 2013, 805-814.

[12] Köse, Ö., On space curves of constant breadth, Doğa Tr. J. Math., 10 (1986), no. 1, 11-14.

[13] Mağden A., Köse, Ö., On the curves of constant breadth in space, Turkish J. Math., 21 (1997), no. 3, $277-284$.

[14] Mellish, A. P., Notes on Differential Geometry, Annals of Math., 32 (1) (1931), 181-190.

[15] Önder, M., Kocayiğit, H., Candan, E., Differential Equations Characterizing Timelike and Spacelike Curves of Constant Breadth in Minkowski 3-spaceJ. Korean Math. Soc. 48 (2011), No. 4, pp. 849-866.

[16] Reuleaux, F., The Kinematics of Machinery, Trans. By A.B.W. Kennedy, Dover, Pub. Nex York, (1963).

[17] Ross, S.L., Differential Equations, John Wiley and Sons, Inc., New York, (1974), 440-468.

[18] Sezer, M., Differential equations characterizing space curves of constant breadth and a criterion for these curves, Turkish, J. of Math. 13 (1989), no. 2, 70-78.

[19] Smakal, S., Curves of constant breadth, Czechoslovak Mathematical Journal, 23 (1973), no. 1, 86-94.

[20] Struik, D.J., Differential Geometry in the Large, Bull. Amer. Math. Soc., 37 (1931), 49-62.

[21] Tanaka, H., Kinematics Design of Com Follower Systems, Doctoral Thesis, Columbia Univ., (1976). 